\title{
Angular Distributions of Discrete Mesoscale Mapping Functions
}

\author{
Krzysztof KROSZCZYŃSKI \\ Military University of Technology, Faculty of Civil Engineering and Geodesy, \\ Warszawa, Poland; e-mail: kkroszczynski@wat.edu.pl
}

\begin{abstract}
The paper presents the results of analyses of numerical experiments concerning GPS signal propagation delays in the atmosphere and the discrete mapping functions defined on their basis. The delays were determined using data from the mesoscale non-hydrostatic weather model operated in the Centre of Applied Geomatics, Military University of Technology. A special attention was paid to investigating angular characteristics of GPS slant delays for low angles of elevation. The investigation proved that the temporal and spatial variability of the slant delays depends to a large extent on current weather conditions.
\end{abstract}

Key words: slant delay, mapping function, mesoscale model, anisotropy.

\section{INTRODUCTION}

Atmospheric refraction plays an important role in precise determination of position by means of Global Positioning System (GPS) techniques. The influence of atmospheric pressure, temperature, and first of all humidity slows the GPS signal down, diffuses it and changes its propagation direction. In consequence, these factors cause the propagation time between the satellite and the receiver to be longer than in the vacuum. These delays, called slant tropospheric delays or corrections, are mainly related with the lower layers of the atmosphere where weather phenomena occur. Since the GPS waves are non-dispersive in the troposphere, the slant delays cannot be eliminated 
and they appear in the observational equations as additional unknowns. In a specific measurement epoch there are as many delays as there are observed GPS satellites. From the computational point of view, this situation is unfavourable and it results in multiplication of the number of the unknowns and usually in a bad conditioning (peculiarity) of the observation matrix. In practice, in order to reduce the number of the unknowns, the slant delays are expressed using zenithal delays (common for all satellites) computed subsequently with other geodesic parameters in the estimation process of observation systems solutions. The relation between these delays is realized using mapping functions dependent on the direction of the satellites observations. Just a few years ago, the available Bernese (Dach et al. 2007) and GAMIT-GLOBK (King and Bock 2005) software packages used mainly the Niell mapping functions (Niell 1996). The coefficients of these functions were determined on the basis of the solutions of the GPS waves propagation equation, the so-called eikonal equation, using selected profiles of the standard atmosphere model and verified using upper air sounding data. This indirect way of determining the slant delays, due to neglecting the current weather conditions, is burdened with a large systematic error, especially for satellites at low elevation angles. For this reason, operational possibility to determine the mapping functions using meteorological data from global weather predicting models was brought to attention (Niell 2000). The research presented in Boehm et al. (2006) was a continuation of Niell's work, and the European Centre for Medium-Range Weather Forecasts (ECMWF) model was used for this. The discrete mapping functions were defined for the global network and the international IGS services (International GNSS Service), IVS (International VLBI Service), and IDS (International DORIS Service). Introducing these mapping functions significantly improved the results of the satellite geodesic techniques and made it possible to realize the precise positioning of a single point (PPP) with sub-centimetre accuracy (Urquhart et al. 2011). Data from the mesoscale non-hydrostatic Coupled Ocean/Atmosphere Mesoscale Prediction System - Naval Research Laboratory (COAMPS-NRL; Hodur 1997, Bosy et al. 2010) model are used for the research presented in this paper. This model, unlike the global ones, due to better spatial resolutions of the computational grids, enables to monitor the temporal and spatial variability of the meteorological elements fields with better effectiveness, especially the humidity which is the main cause of difficulties encountered in GPS signal delay modelling. The values of the delays, especially for low elevation angles, may have significant influence on the solutions of the observation systems. For this reason, the proper determination of the delays is extremely important for accuracy of spatial geodesic techniques and it has significant influence on the accuracy of geodesic parame- 
ters computations (e.g., Bogusz et al. 2011, Urquhart et al. 2011, Wielgosz et al. 2012).

\section{SLANT DELAY OF GPS SIGNAL}

The considered slant delay $\Delta \tau^{s}$ is a result of integrating the refraction coefficient $n$ of the atmosphere along the GPS signal path $s$ from the satellite to the receiver (Fig. 1) according to Eq. 1. In vacuum the signal propagates along a straight line while in the atmosphere (according to the Fermat principle) along a curve line. Beside the curvature called the geometric elongation effect $\Delta \tau^{g}$, the signal is first of all slowed down. The slowdown effect (additional elongation) is called the electromagnetic effect $\Delta \tau^{e}$ :

$$
\Delta \tau^{s}=\underbrace{\int_{\text {sat }}^{\text {rec }} n(s) d s-\int_{\text {sat }}^{\text {rec }} 1 d s+}_{\Delta \tau^{e}}+\underbrace{\int_{\text {sat }}^{\text {rec }} 1 d s-\int_{\text {sat }}^{\text {rec }} 1 d l}_{\Delta \tau^{g}},
$$

where $\Delta \tau^{s}$ is the total delay of the signal in the atmosphere (expressed in units of length, e.g., meters), and $d s, d l$ are the differential increments of the $s$ and $l$ (straight line) paths' lengths of the GPS signal from the satellite (sat) to the receiver $(\mathrm{rec})$.

The $\Delta \tau^{g}$ element defines the curvature and represents the geometric delay resulting from the difference in length of the signal paths calculated along the curve and the straight line connecting the receiver and the satellite. The geometric delay $\tau^{g}$ has significant contribution (decimetres) for low elevation satellites; it is less than $1 \mathrm{~cm}$ for elevation angles greater than $15^{\circ}$ and it equals zero in the direction of the zenith (Mendes 1999).

Meteorological parameters generated by the COAMPS mesoscale model, i.e., potential temperature, total pressure, and specific humidity (Figurski et al. 2007, 2009), were used for determining tridimensional refraction fields $n_{x y z}$. The fields were interpolated from the computational space of the mesoscale model (Lambert's Conformal Projection with the Gal-Chen

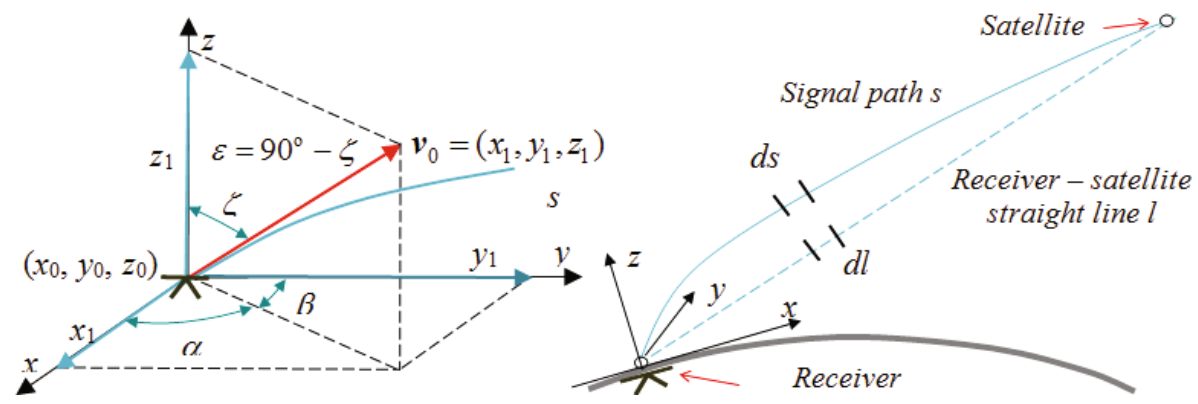

Fig. 1. The geometry of the GPS signal propagation. 
and Somerville's nonlinear vertical coordinate) into the parallel planes of a local topocentric coordinate system defined by the GPS station position (Fig. 1). The Cartesian coordinate system used for realizing the computations simplifies preparation of the algorithm of the eikonal equation called also the ray Eq. 2 written in the form of ordinary differential equations system:

$$
\frac{d}{d s}\left[n(\mathbf{r}) \frac{d \mathbf{r}}{d s}\right]=\nabla n(\mathbf{r}) \rightarrow\left\{\frac{d \mathbf{r}}{d s}=\frac{1}{n(\mathbf{r})} \mathbf{v}, \frac{d v}{d s}=\nabla n(\mathbf{r})\right\},
$$

where $s$ is the path measured along the ray (Fig. 1) and defined by the vector $\mathbf{r}=[x(s), y(s), z(s)]$ with respect to the beginning of the topocentric reference system, $v$ is the vector tangential to the trajectory (ray) at point $\mathbf{r}$, and $n(\mathbf{r})$ is the refraction coefficient dependent on the radius vector $\mathbf{r}$.

The equation system 2 indicates that both the length of the path $s$ and the value of the slant delay $\Delta \tau$ are dependent on the spatial heterogeneousness of the refraction coefficient $n(\mathbf{r})$ defined by the gradient $\nabla n(\mathbf{r})$. The solution of the system 1 was formulated in the form of the following initial-value problem:

$$
\begin{aligned}
& \mathbf{r}=\mathbf{r}_{0} \\
& \mathbf{v}_{0}=\left(d x\left(s_{0}\right) / d s, d y\left(s_{0}\right) / d s, d z\left(s_{0}\right) / d s\right)=\cos \alpha, \cos \beta, \sin \left(90^{\circ}-\zeta\right) .
\end{aligned}
$$

In Eq. $3 \mathbf{r}_{0}=\left[x\left(s_{0}\right), y\left(s_{0}\right), z\left(s_{0}\right)\right]$ is a vector of the GPS station antenna location, $\zeta$ is the zenithal angle, $v_{0}$ is a versor tangential to the trajectory at point $\mathbf{r}_{0}$ which is determined by the directional cosines: $\cos \alpha, \cos \beta, \cos \xi$ defining the direction to a selected point in the space, e.g., the satellite position. In order to determine signal delay of the particular satellite, shooting method (Hobiger et al. 2008) or collocation method (Zus et al. 2012) is used.

\section{THE ALGORITHM OF DETERMINING THE SLANT DELAY}

The path $s$ of the ray and the slant delays $\Delta \tau$ related with it were determined using an iteration algorithm (Figurski et al. 2007, 2009). For every GPS station it uses atmospheric refraction fields defined in the topocentric systems (Fig. 1) on rectangular grids interpolated from irregular grids (of spatial resolution of 13 and $4.3 \mathrm{~km}$ in the Lambert's Conformal Projection) of the mesoscale model. The distribution of distances between the computational planes (levels) of these grids is not uniform. Distributions similar to that presented in Rocken et al. (2001) were used for the research. This solution saves the operational memory of the computer and shortens the time of computing while maintaining appropriate accuracy of the obtained values of the slant delay. It is also compatible with the distribution of atmospheric fields in real atmosphere, in the altitude reference system, and in mesoscale mod- 
els. In the grids considered here, the cells are cuboids (Fig. 2) with different heights $d h$ and constant lengths of the sides $d x, d y$ equal to the distances between the nodes of the mesoscale model cells. The program for local threeline interpolation is introduced because of the applied procedures of solving the equations system 1 and thus the necessity of computing the values of refraction in any point of the computational cell (Fig. 2).

$$
\begin{aligned}
n_{x y z}= & n_{000}(1-x)(1-y)(1-z)+n_{100} x(1-y)(1-z)+n_{010}(1-x)(1-y) \\
& +n_{101} x(1-y) z+n_{011}(1-x) y z+n_{110} x y(1-z)+n_{111} x y z .
\end{aligned}
$$

The variables $(x, y, z)$ in Eq. 4 are normalized. The normalization is equivalent to an auxiliary transformation of the cuboids cells into unitary cubes (Fig. 2).

Polynomial form of the function $n_{x y z}$ simplifies determination of gradient components $n_{x y z}$ vector which are necessary for integrating realization procedure for the differential equations system 2 of the ray. The procedure determines the trajectory, i.e., the GPS signal path. The classical constant step Runge-Kutta method of the fourth order (Ralson 1995) was used here. Identifications of the cells of the computations grid in which the determined point of the trajectory is placed (Fig. 2), is an important phase of the algorithm. For irregular grids, this identification is usually a complex process and computationally expensive. However, it is simple for the used rectangular grids. In this case, the cells are identified by the $(i, j, k)$ indexes defined by:

$$
(j, j, k)=\left(\text { floor }\left(\left(x_{0}+x^{*}\right) / d x\right) \text {, floor }\left(\left(y_{0}+y^{*}\right) / d y\right) \text {, floor }\left(\left(z_{0}+z^{*}\right) / d h\right)\right),
$$

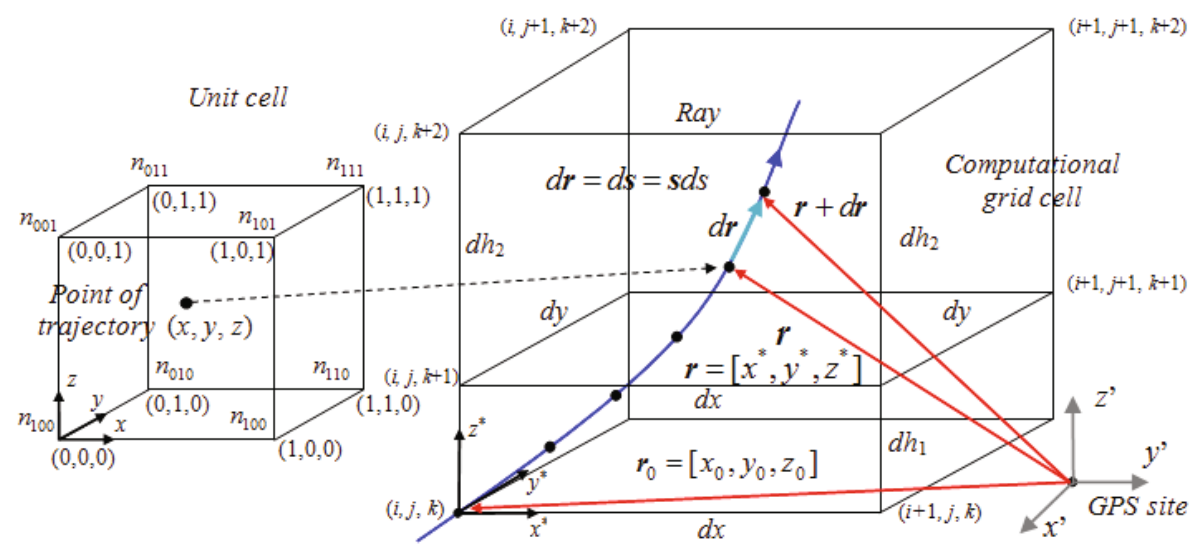

Fig. 2. Computational algorithm illustration - the three-line interpolation. 
where "floor" is a function rounding the numbers down, and $\mathbf{r}_{0}=\left[x_{0}, y_{0}, z_{0}\right]$ is the vector connecting the centre of the topocentric system with the local system $\left(x^{*}, y^{*}, z^{*}\right)$ of the current cell of the grid (Fig. 2).

Procedure 5 may also be applied to regular spherical grids. The refraction value and the gradient 4 are computed for a new point of the trajectory after determining the point and identifying the cells of the grid (Fig. 2). The new position is then determined by means of the procedure of integration. The iteration process is continued until the modelled atmosphere is left. Recording the coordinates of the points of the trajectory and their related values of refraction $n_{x y z}$ enables to compute the slant delay value $\Delta \tau^{s}$ (Eq. 1).

\section{NUMERICAL EXPERIMENTS}

\subsection{Meteorological conditions}

For the conducted basic research, every forecast from the model may be considered as the real state. However, having in mind future provision of mesoscale zenithal $\Delta \tau^{z}$ and slant $\Delta \tau^{s}$ delays for the users, as well as mapping functions $m=\Delta \tau^{s} / \Delta \tau^{z}$ determined on their basis, we have to be aware that the question of confronting the model results with the actual conditions, i.e., appropriate monitoring and validation of the mesoscale model performance, will be inevitable. For this reason, investigating propagation of the GPS waves was related with periods in which the state of the atmosphere determined by the COAMPS mesoscale model correctly reflected the real atmospheric conditions.

To quantitatively verify the results, MSG 2 (Meteosat Second Generation) satellite images from the archives of the Dundee University, http://www.sat.dundee.ac.uk/, were used. Cloud systems related with atmospheric precipitation are observed in the IR7 (8.3-9.1 $\mu \mathrm{m})$ images (Fig. 3). The satellite images are presented in the COAMPS model projection. It is observed that areas of precipitation and related cloud systems generated by the model are correlated with the cloud systems observed in the images. It is, among others, an effect of the observations data assimilation process which determines the initial conditions of the weather forecast. Partial quantitative verification of the atmosphere state, especially concerning the surface fields in the area of Poland, may be conducted using data provided by synoptic stations of the Institute of Meteorology and Water Management. If all sites were equipped with automatic systems measuring basic meteorological parameters, it would provide excellent auxiliary material from the Active Geodetic Network European Position Determination System (ASG EUPOS) network. Currently, EUREF Permanent Network (EPN) and the WAT1 sites are equipped with such stations. 


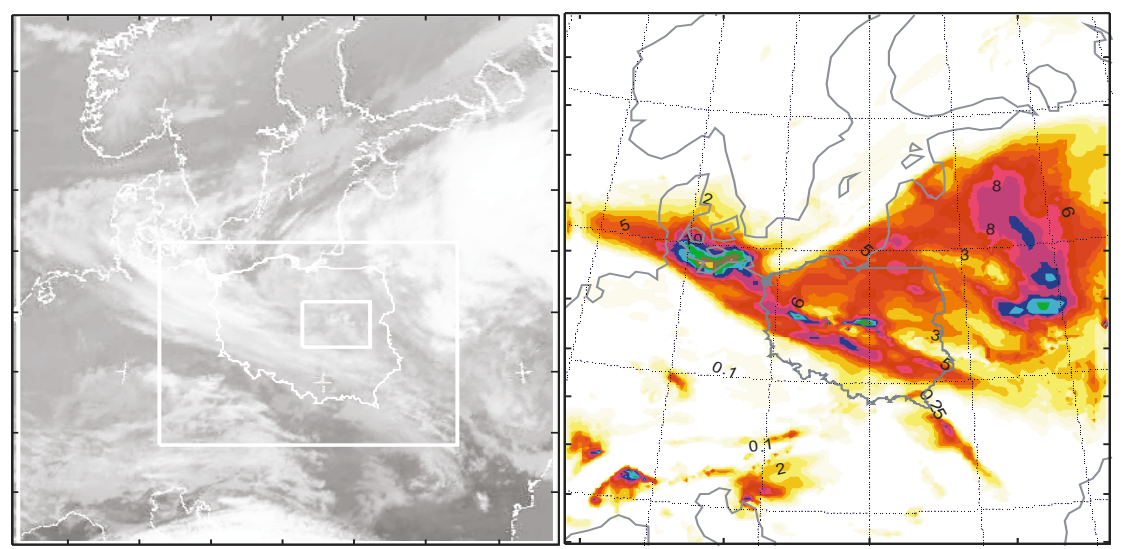

Fig. 3. Comparison of the cloud cover fields observed in the MSG 2 satellite images in the IR7 channel and the forecasted fields of precipitation for the $13 \mathrm{~km}$ computational grid of the COAMPS model at 6.00 on 17 April 2008.

\subsection{The process of scanning the atmosphere}

The process of scanning the atmosphere generated by the COAMPS mesoscale model was used in order to investigate the character of the slant delay spatial distribution (Figurski et al. 2009). The slant delays were determined according to the scheme presented in Fig. 4 for elevation angles $\varepsilon$ within the range of $\left[3^{\circ}, 10^{\circ}\right]$ and $\left[15^{\circ}, 90^{\circ}\right]$ with increments of $1^{\circ}$ and $5^{\circ}$, respectively, and for azimuths $\alpha$ within the range of $\left[0^{\circ}, 350^{\circ}\right]$ with increments of $10^{\circ}$. It takes into consideration $24 \times 36=864$ directions.

Such sampling of the space enables us to obtain appropriately accurate interpolated values of the delay for any direction. Due to the minimum value of the elevation angle, $\varepsilon=3^{\circ}$, the computations could not be realized for all
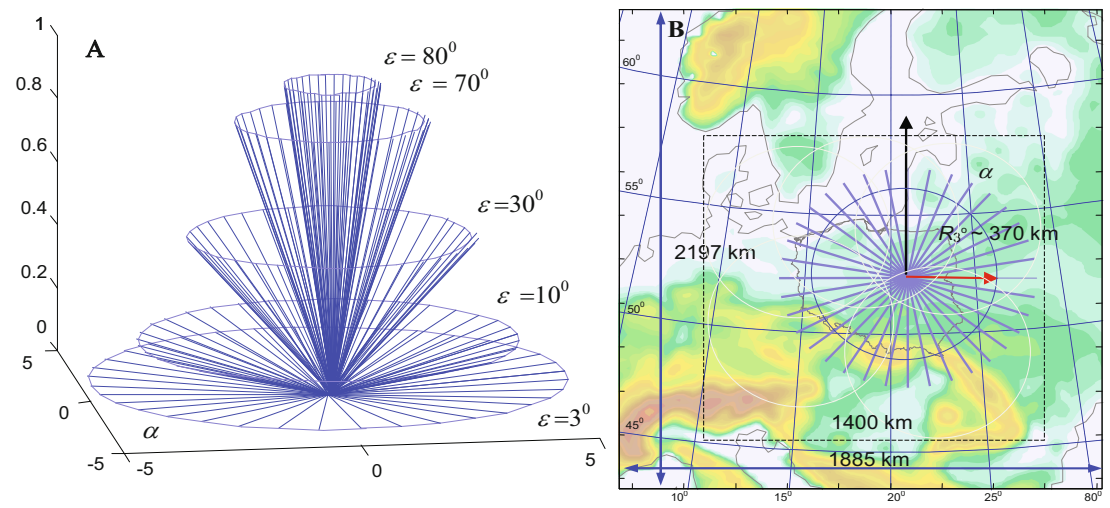

Fig. 4. The spatial process of scanning the atmosphere. 

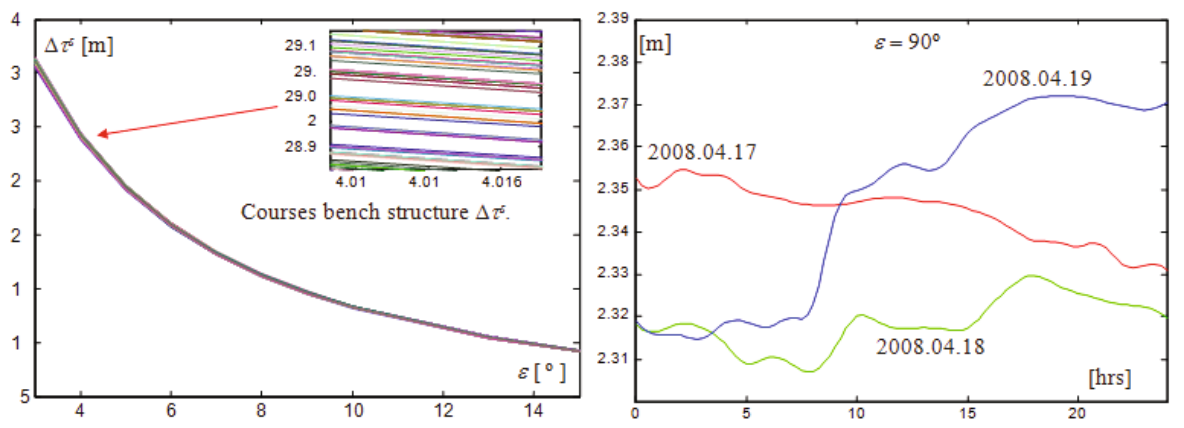

Fig. 5. Slant delay as a function of the elevation angles for fixed azimuth values and mesoscale model forecast time. Daily course of zenithal delay for the WAT1 site.

points of the mesoscale model space. For example, the area of 1885 by $2197 \mathrm{~km}$ (13 km grid), as presented in Fig. 4B, is appropriate for determining the full distributions of the slant delay $\Delta \tau^{s}$ for the rays inclined at $3^{\circ}$ with respect to the plane of the horizon for all points belonging to the territory of Poland (except for those that are subject to visibility problems). The lengths of the rays projections $R_{\mathrm{z}}$ (Eq. 6) for the elevation angles $\varepsilon$ (Fig. 4B) are defined by the $(x, y)$ coordinates of the intersection of the sphere of $a+h$ radius and the straight line defined by the equation: $y=x \times \operatorname{tg} \alpha$ and crossing the centre of the topocentric coordinate system:

$$
R_{z}=\operatorname{arctg}(x / z) \leftarrow\left(y=x \times \operatorname{tg} \varepsilon+a, x^{2}+y^{2}=(a+h)^{2}\right),
$$

where $a=6371.229 \mathrm{~km}$, and $h=30 \mathrm{~km}$ is the height of the COAMPS atmospheric model.

For $\varepsilon=3^{\circ}$ Eq. 6 provides $R_{\varepsilon=3^{\circ}} \approx 370 \mathrm{~km}$. The $R_{\varepsilon}$ parameter value usually influences the choice of the spatial resolution of the mesoscale model grid. Figure 4B shows that a square of the side length of about $1400 \mathrm{~km}$ is sufficient to determine $\Delta \tau^{s}$ for $R_{\varepsilon=3^{\circ}}$. The courses of slant delays $\Delta \tau^{s}$ presented in Fig. 5 as functions of the elevation angles for fixed azimuth values, mesoscale model forecast time and daily zenithal delays $\Delta \tau^{z}$ are the results of the computations.

\subsection{Mesoscale mapping functions}

Using the plots of Fig. 5, complete discrete mapping functions $m_{c}$ may be derived which are dependent on GPS station position $x=(\lambda, \phi, h)$, time $t$, elevation $\varepsilon$, and azimuth $\alpha$ angles determined by the process of scanning the mesoscale model atmosphere: 


$$
\begin{aligned}
m_{c} & =\left(\varepsilon_{j}, \alpha_{j}, t_{k}, x\right)=\frac{\Delta \tau^{s}\left(\varepsilon_{j}, \alpha_{j}, t_{j}, x\right)}{\Delta \tau^{s}\left(\varepsilon=90^{\circ}, t_{k}, x\right)}=\frac{\Delta \tau^{s}\left(\varepsilon_{j}, \alpha_{j}, t_{j}, x\right)}{\Delta \tau^{z}\left(t_{k}, x\right)}, \\
i & =\left[3^{\circ}, 4^{\circ}, \ldots, 9^{\circ}\right] \cup\left[10^{\circ}, 15^{\circ}, \ldots, 90^{\circ}\right], j=0^{\circ}, 10^{\circ}, 20^{\circ}, \ldots, 350^{\circ}, k=1,2, \ldots, 24 \mathrm{~h},
\end{aligned}
$$

where $\Delta \tau^{z}=\Delta \tau^{s}\left(\varepsilon=90^{\circ}\right)$ is the zenithal delay.

In case of investigating the influence of water vapour content on GPS signals propagation or a reciprocal task, e.g., GPS tomography of the atmosphere using the complete mapping function $m_{c}$, it is possible to extract its wet $m_{w}$ and hydrostatic $m_{h}$ parts. Determination of $m_{w}$ and $m_{h}$ (Rocken et al. 2001, Urquhart et al. 2011) is realized using the following relations:

$$
\begin{aligned}
& n=n_{w}+n_{h} \rightarrow\left(\Delta \tau_{w}^{s}, \Delta \tau_{w}^{z}\right) \\
& \left(\Delta \tau_{w}^{s}, \Delta \tau_{w}^{z}\right) \rightarrow\left(m_{w}=\Delta \tau_{w}^{s} / \Delta \tau_{w}^{z}, m_{h}=\Delta \tau_{h}^{s} / \Delta \tau_{h}^{z}\right),
\end{aligned}
$$

where $\Delta \tau_{h}^{s}, \Delta \tau_{w}^{s}, \Delta \tau_{h}^{z}, \Delta \tau_{w}^{z}, n_{w}, n_{h}$ are dry and wet parts of the slant $\Delta \tau^{s}$ and zenithal $\Delta \tau^{z}$ delays and of the refraction coefficient $n$.

For the $t=0$ epoch, the $m_{c}$ functions are computed using the analysis of meteorological fields, while for $t \neq 0$ using forecasts obtained from the mesoscale model. The analysis is based on real data which have to be collected from the meteorological data exchange network for the main synoptic times $(00,06,12,18$ UTC). The delay related with it causes that operational application of $m_{c}$ is feasible for ultra-short, e.g., 6-hourly weather forecasts. Equation 8 shows that for the measurement epoch $t$, the $m_{c}$ function differs from the $\Delta \tau^{s}$ by the multiplication factor which is a reciprocal of the $\Delta \tau^{z}$ delay. Due to relatively small values of changes of $\Delta \tau^{z}$ (Fig. 5), investigating $m_{c}$ or $\Delta \tau^{s}$ in the range of low elevation angles $\varepsilon$ is practically equivalent. For this reason, amplitudes $m_{c}$ and $\Delta \tau^{s}$ are proportional, and their angular characteristics are identical. The functions described by Eq. 8 vary from the simplified ones routinely used in GPS analyses by anisotropy of the spatial distribution. The simplifications are first of all related to the assumed independence of azimuth $\alpha$. The research presented below indicates that such an assumption is justified only for appropriately large elevation angles assumed in practice as the cut-off angles $\left(\varepsilon>10^{\circ}\right)$.

\section{ANALYSIS OF SLANT DELAY DISTRIBUTIONS}

Investigating the mapping functions 7 is not simple because of their multidimensionality. Therefore, the analysis method of projections on sub-spaces of various combinations of the $\varepsilon, \alpha, t, x$ parameters was used for this purpose. For example, the projection on the $\varepsilon, \alpha$ sub-space is equivalent to fixing the position $x$ of the GPS site and fixing the time $t$ of observation. The 
first plot (Fig. 5) illustrates the relation 9 between the slant delay $\Delta \tau^{s}$ and the elevation angle $\varepsilon$ for constant value of azimuth $\alpha$ and time $t$ for the WAT1 reference site. The other plot (Fig. 5) presents one-dimensional temporal courses of the zenithal delay described by Eq. 10. They reflect, among others, the changes of meteorological conditions (e.g., air humidity) above the observation point.

$$
\begin{gathered}
\Delta \tau^{s}=\Delta \tau^{s}(\varepsilon, \alpha=\text { const }, t=\text { const }, x), \\
\Delta \tau^{s}=\Delta \tau^{s}\left(\varepsilon=90^{\circ}, \alpha=\text { const }, t, x=x_{\text {WAT1 }}\right) .
\end{gathered}
$$

From a practical point of view, the sets of values of function 7 for elevation and azimuth angles determined by the varying in time position of GPS satellites are important for the GPS solutions. Generally, the slant delays $\Delta \tau^{s}$ for a selected GPS station are determined by means of fast methods of ray tracking according to the scanning scheme presented in Fig. 4. Forecasted refraction fields of the mesoscale model determined in one hour intervals are used in the computations. Tables 1 and 2 contain examples of angular distributions of slant delays for 17 and 19 April 2008, averaged with respect to azimuth and time (day).

The sets include the minimum $\Delta \tau_{\min }^{s}(\varepsilon)$, maximum $\Delta \tau_{\max }^{s}(\varepsilon)$, and average $\overline{\Delta \tau^{s}(\varepsilon)}$ values and the standard deviations of the delays for the elevation angles $\varepsilon$ considered in the computations. They are determined for 900element samples of data. The averaging $\overline{\Delta \tau^{s}(\varepsilon)}$ was done with respect to a set of azimuths $\alpha$ from the range of $\left(0^{\circ}, 360^{\circ}\right)$ with $10^{\circ}$ increments. Analysis of the results obtained for two selected days indicates that for the elevation angle $\varepsilon=3^{\circ}$, the azimuth differences of the delays are up to $1.1 \mathrm{~m}$ (on 17 April) and $1.6 \mathrm{~m}$ (on 19 April). They decrease with increasing $\varepsilon$, reaching 14 and $28 \mathrm{~cm}$ for the elevation angle of $\varepsilon=15^{\circ}$. Additional comparison of estimated values of standard deviations $\sigma_{\Delta t(\varepsilon)}$ shows that the distribution for 19 April has greater spatial variability than the distribution for 17 April (Fig. 6). Therefore, it may be concluded that the GPS weather defined by the spatial variability and irregularity was worse for the meteorological conditions observed at 00 UTC on the second of the considered days (Table 2). It is interesting that the conclusion might be opposite if the average values $\overline{\Delta \tau^{s}(\varepsilon, \alpha)}$ were considered alone. In this case, the maximum difference is only $5 \mathrm{~cm}$. The presented example illustrates the dissimilarity of the anisotropic mesoscale mapping functions developed in the research and the isotropic NMF or VMF1 type functions (Niell 1996, Boehm et al. 2006). 
Table 1

Angular distribution of slant delays for the WAT1 reference station, 17 April 2008, 00 UTC

\begin{tabular}{|c|c|c|c|c|}
\hline$\varepsilon$ & $\Delta \tau_{\min }^{s}(\varepsilon)$ & $\overline{\Delta \tau^{s}(\varepsilon)}$ & $\Delta \tau_{\max }^{s}(\varepsilon)$ & $\sigma_{\Delta \tau(\varepsilon)}$ \\
{$[\mathrm{m}]$} & {$[\mathrm{m}]$} & 36.45 & 0.21 \\
\hline $3^{\circ}$ & 35.35 & 36.00 & 29.46 & 0.14 \\
$4^{\circ}$ & 28.79 & 29.16 & 24.62 & 0.11 \\
$5^{\circ}$ & 24.11 & 24.39 & 21.09 & 0.09 \\
$6^{\circ}$ & 20.69 & 20.91 & 18.44 & 0.07 \\
$7^{\circ}$ & 18.10 & 18.29 & 16.37 & 0.06 \\
$8^{\circ}$ & 16.08 & 16.24 & 14.73 & 0.06 \\
$9^{\circ}$ & 14.47 & 14.61 & 13.39 & 0.05 \\
$10^{\circ}$ & 13.16 & 13.29 & 10.56 & 0.04 \\
$13^{\circ}$ & 10.38 & 10.48 & 9.28 & 0.03 \\
$15^{\circ}$ & 9.14 & 9.22 & &
\end{tabular}

Table 2

Angular distribution of slant delays for the WAT1 reference station, 19 April 2008, 00 UTC

\begin{tabular}{|c|c|c|c|c|}
\hline$\varepsilon$ & $\Delta \tau_{\min }^{s}(\varepsilon)$ & $\overline{\Delta \tau^{s}(\varepsilon)}$ & $\Delta \tau_{\max }^{s}(\varepsilon)$ & $\sigma_{\Delta \tau(\varepsilon)}$ \\
& {$[\mathrm{m}]$} & {$[\mathrm{m}]$} & {$[\mathrm{m}]$} & {$[\mathrm{m}]$} \\
\hline $3^{\circ}$ & 35.00 & 35.96 & 36.60 & 0.38 \\
$4^{\circ}$ & 28.53 & 29.14 & 29.61 & 0.29 \\
$5^{\circ}$ & 23.93 & 24.39 & 24.75 & 0.24 \\
$6^{\circ}$ & 20.53 & 20.91 & 21.22 & 0.21 \\
$7^{\circ}$ & 17.96 & 18.29 & 18.55 & 0.18 \\
$8^{\circ}$ & 15.96 & 16.25 & 16.48 & 0.16 \\
$9^{\circ}$ & 14.36 & 14.62 & 14.82 & 0.14 \\
$10^{\circ}$ & 13.06 & 13.29 & 13.47 & 0.13 \\
$13^{\circ}$ & 10.31 & 10.49 & 10.63 & 0.10 \\
$15^{\circ}$ & 9.07 & 9.23 & 9.35 & 0.09 \\
\hline
\end{tabular}

The plots of the delay differences and their average values for each hour of the forecast:

$$
\Delta \tau_{r}^{s}=\Delta \tau^{s}-\overline{\Delta \tau^{s}}, \Delta \tau^{s}=\Delta \tau^{s}\left(\varepsilon, \alpha, t, x_{\mathrm{WAT} 1}\right), t=0,1, \ldots, 24 \mathrm{~h}
$$

are presented in Fig. 6 , where $x_{\mathrm{WAT} 1}$ is the WAT1 reference site position vector.

Application of the differences presents in a clearer way the dependence of delays on the elevation $\varepsilon$ and azimuth $\alpha$ angles. Observing them in the abso- 

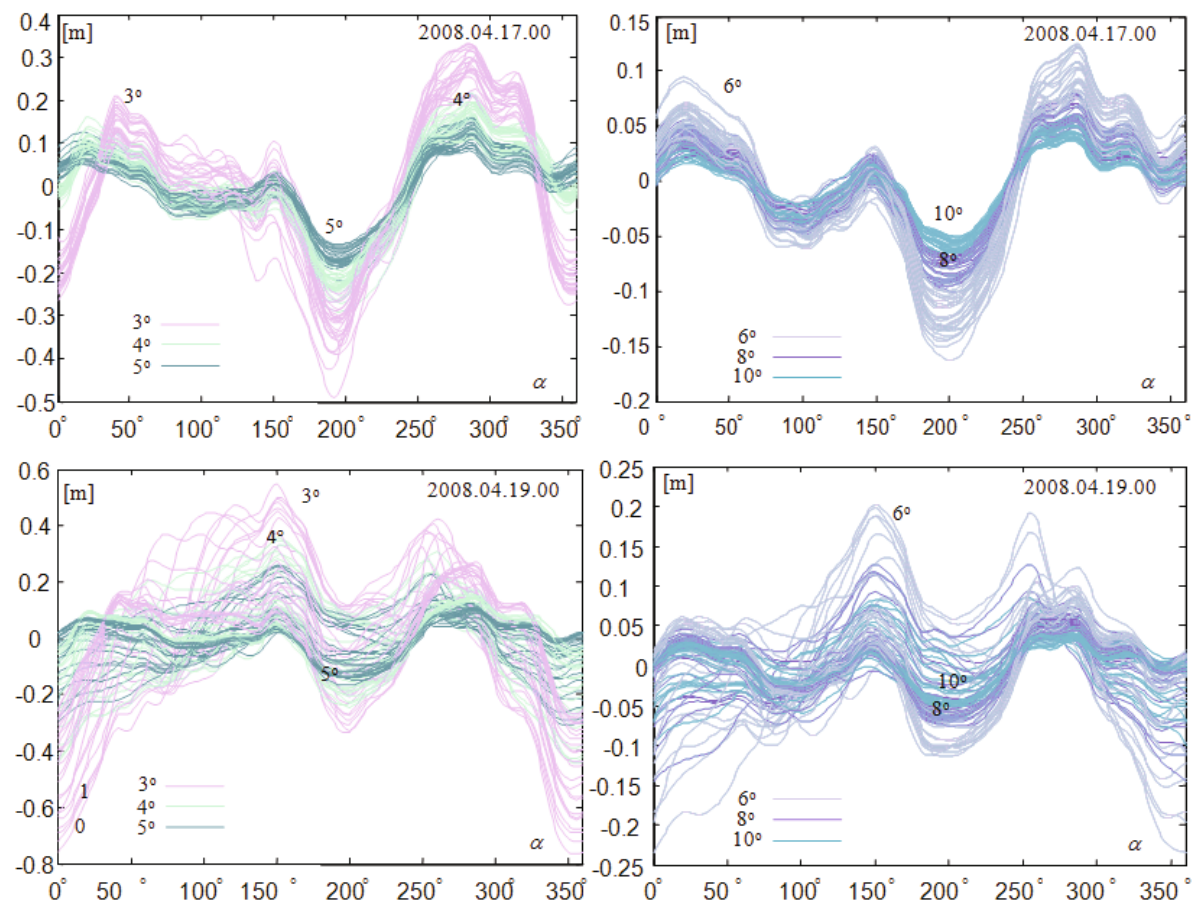

Fig. 6. Differences of slant delays as functions of azimuth and elevation angles for the WAT1 site.

lute courses is more difficult because the disturbances (differences) amplitude for the elevation angle of $5^{\circ}$ is about 50 times smaller than the average value. The plots of Fig. 6 may also be presented in the polar form which is more comfortable for analysis.

Figures 7-12 present azimuth distributions of differences of slant delays $\Delta \tau_{r}^{s}$ for the WAT1 site for elevation angles $\varepsilon=3^{\circ}, 4^{\circ}, 5^{\circ}$. They were constructed using the following equations:

$$
\begin{aligned}
& x=\left|\Delta \tau_{r}^{s}(\cdot, \alpha)\right| \cos \alpha, \quad y=\left|\Delta \tau_{r}^{s}(\cdot, \alpha)\right| \sin \alpha, \\
& \Delta \tau_{r}^{s}(\cdot, \alpha)=\Delta \tau^{s}(\cdot, \alpha)-\overline{\Delta \tau^{s}(\cdot, \alpha)}, \\
& \Delta \tau^{s}(\cdot, \alpha)=\Delta \tau^{s}\left(\varepsilon \in\left\{3^{\circ}, 4^{\circ}, 5^{\circ}\right\}, \alpha, t \in\{1,24\}, x_{\mathrm{WAT} 1}\right),
\end{aligned}
$$

where $\Delta \tau^{s}(\cdot, \alpha), \overline{\Delta \tau^{s}(\cdot, \alpha)}$ is the discrete course and its average with respect to azimuth (Fig. 6) for each hour $(t=0,1, \ldots, 24)$ of the daily forecast; $x, y$ are coordinates of radius vectors of the $\left|\Delta \tau_{r}^{s}(\cdot, \alpha)\right|$ lengths determined for the set of azimuths: $\alpha \in\left[0^{\circ}, 360^{\circ}\right)$. 

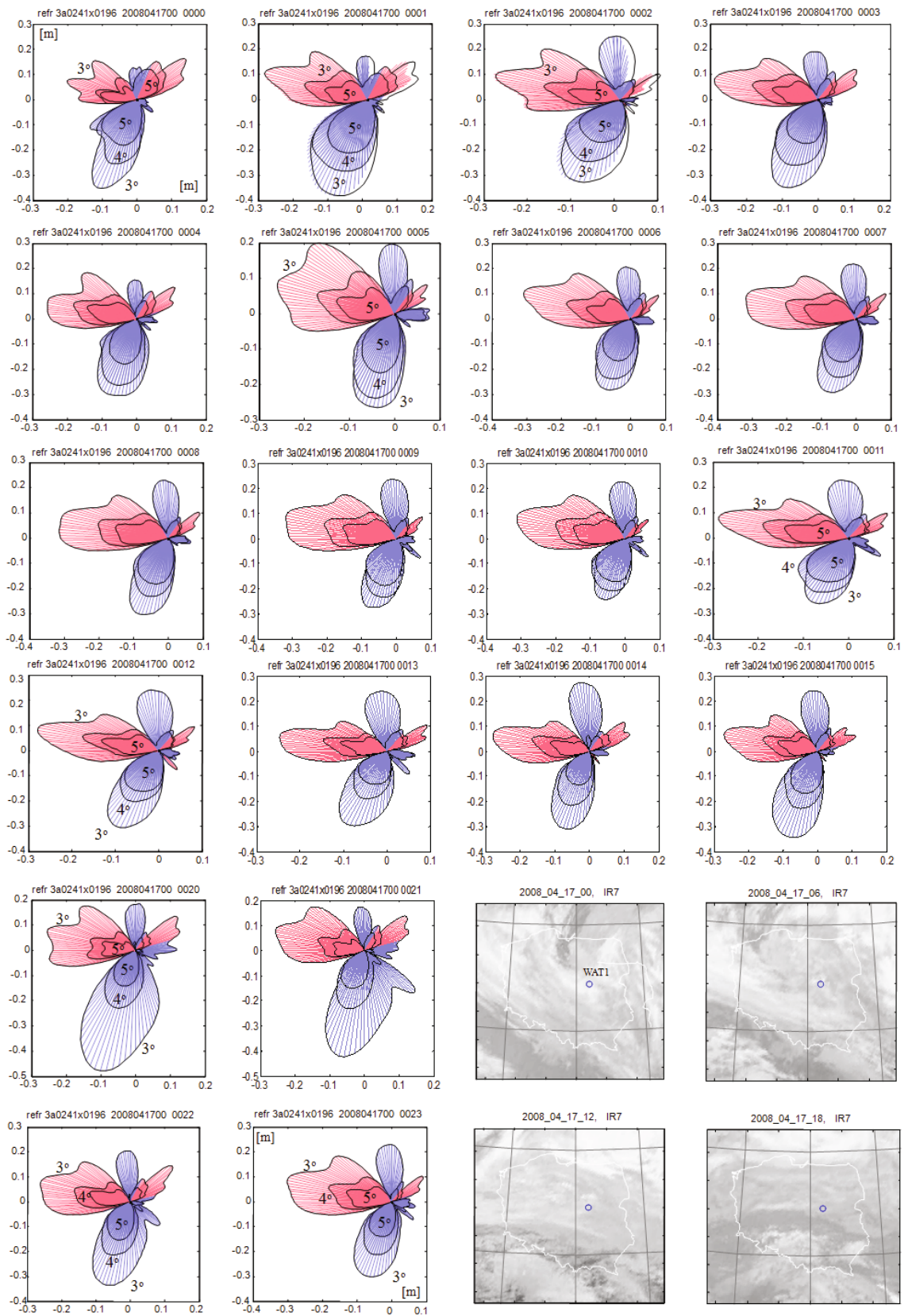

Fig. 7. Anisotropic distributions of the differences of the slant delay $\Delta \tau_{r}^{s}=\Delta \tau^{s}-\overline{\Delta \tau^{s}}\left(\overline{\Delta \tau^{s}}\right.$ is the average with respect to the azimuth) for a daily forecast on 17 April 2008. The synoptic situation is presented in MSG 2 IR7 satellite images. Units: $[\mathrm{m}]$. 

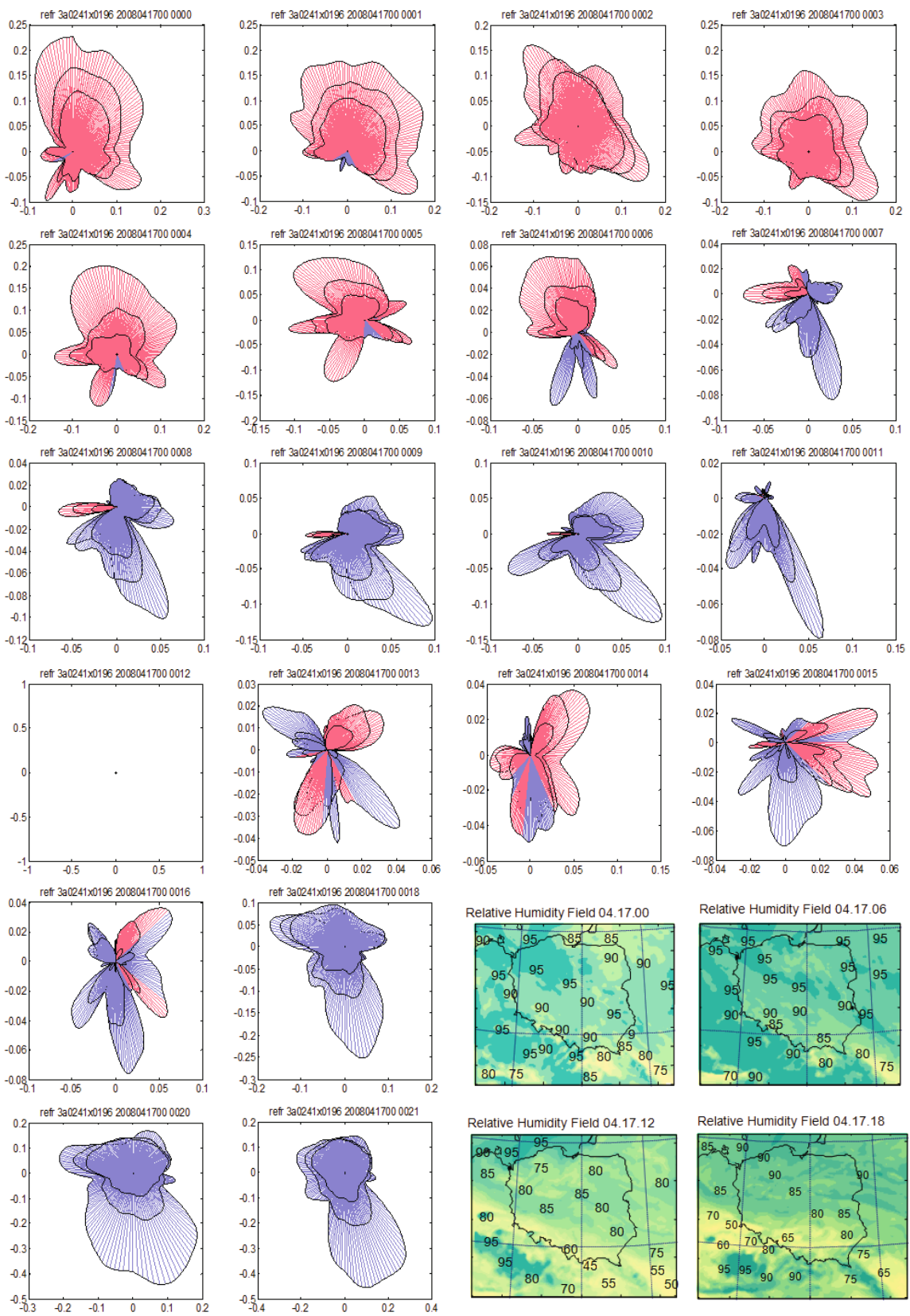

Fig. 8. Azimuth distributions of the differences of the slant delay $\Delta \tau_{r}^{s}=\Delta \tau^{s}-\Delta \tau^{s}\left(\cdot, t_{12}\right) \quad\left(\Delta \tau^{s}\left(\cdot, t_{12}\right)\right.$ is the course for the 12 hour forecast $)$ on 17 April 2008. The synoptic situation is presented in relative humidity fields. Units: [m]. 

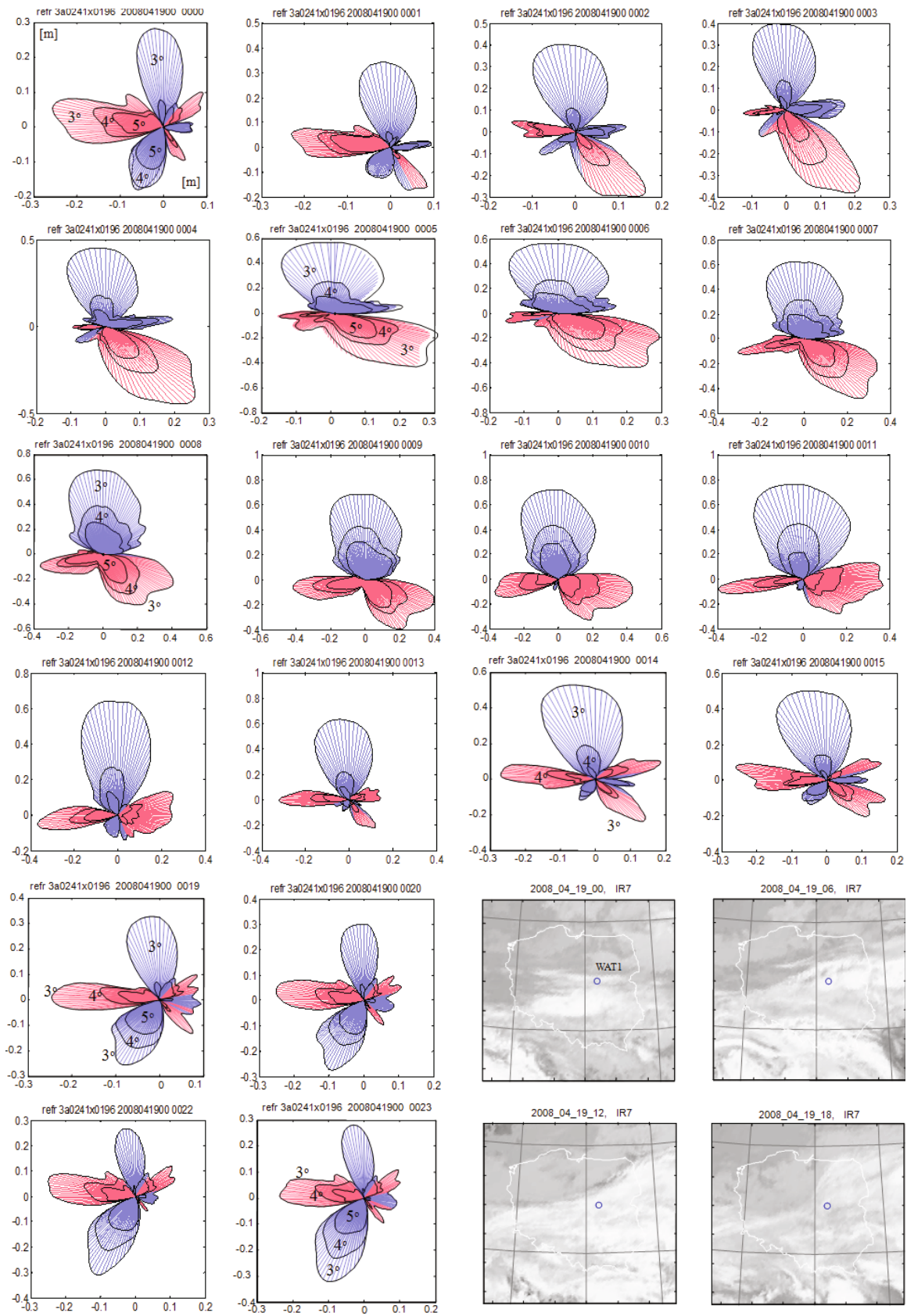

Fig. 9. Distributions of the differences $\Delta \tau_{r}^{s}=\Delta \tau^{s}-\overline{\Delta \tau^{s}}$ of the slant delay for elevation angles of $3^{\circ}, 4^{\circ}, 5^{\circ}$ at subsequent hours of the daily forecast on 19 April 2008 for the synoptic situation presented in MSG 2 IR7 satellite images. Units: [m]. 

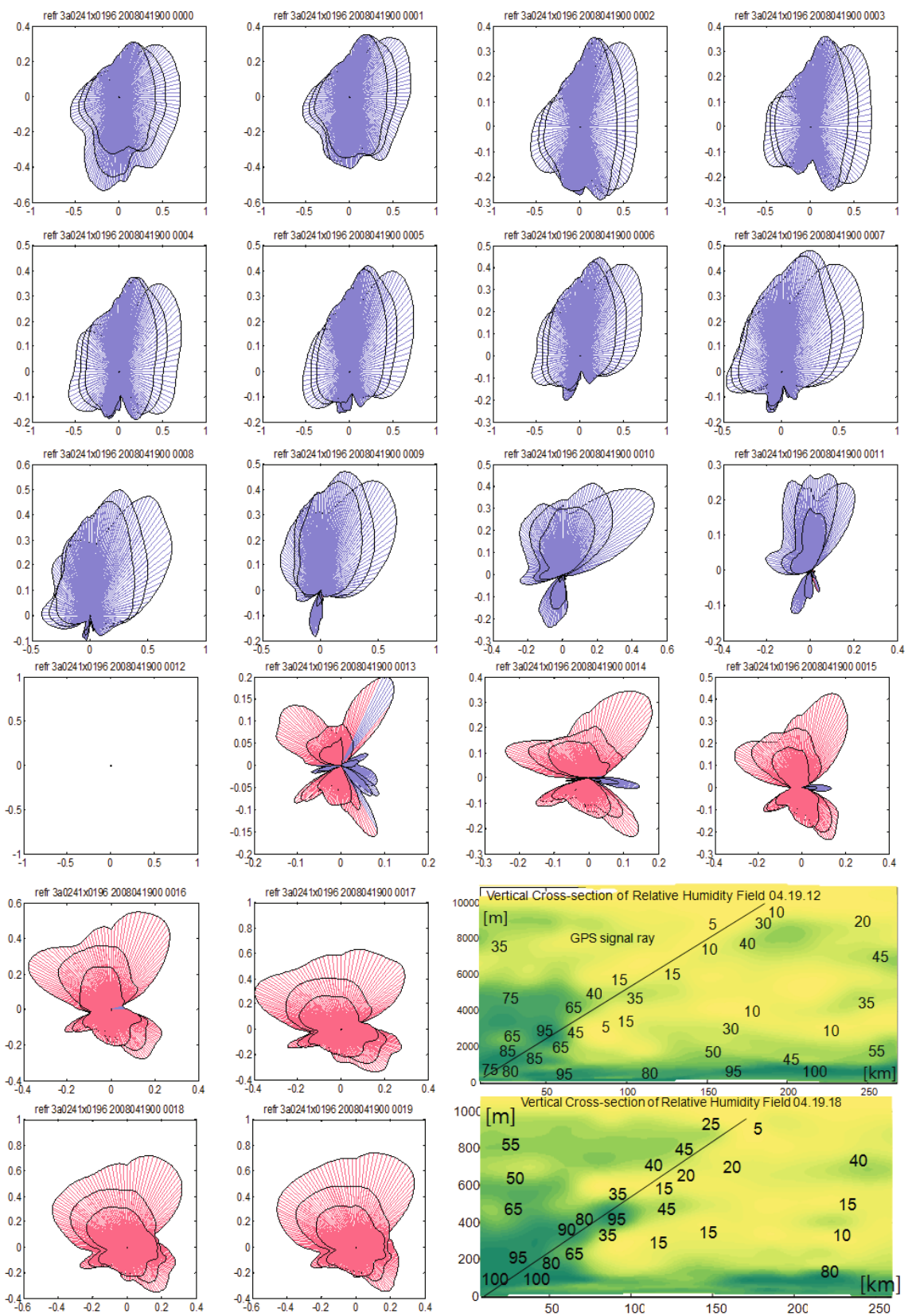

Fig. 10. Anisotropic distributions of the differences of the slant delay $\Delta \tau_{r}^{s}=\Delta \tau^{s}-\Delta \tau^{s}\left(\cdot, t_{12}\right)$ at subsequent hours of the daily forecast on 19 April 2008. The GPS signal rays for the elevation angle of $3^{\circ}$ are superimposed over vertical cross-sections of the humidity field. Units: [m]. 

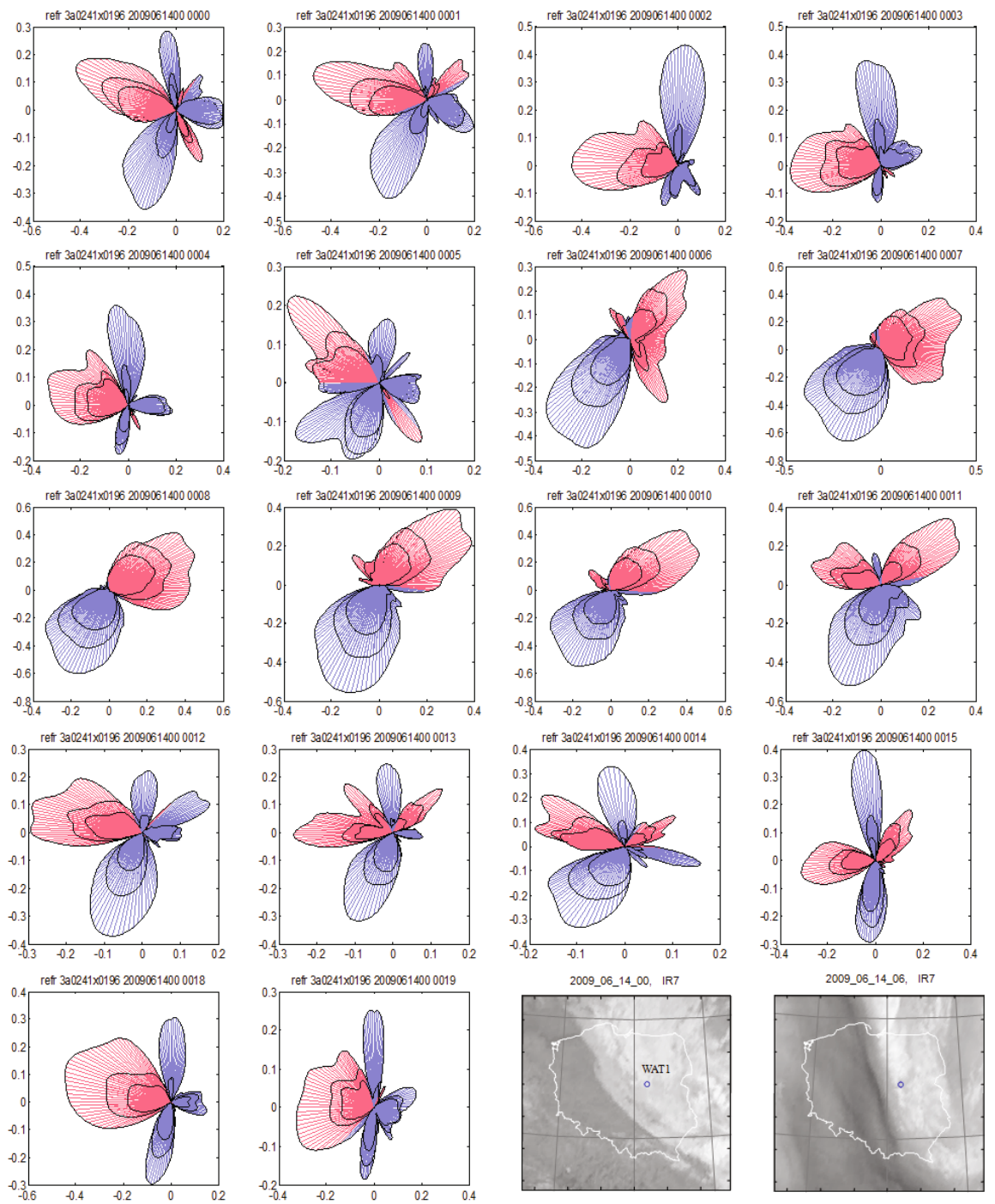

2009_06_14_06, IR7
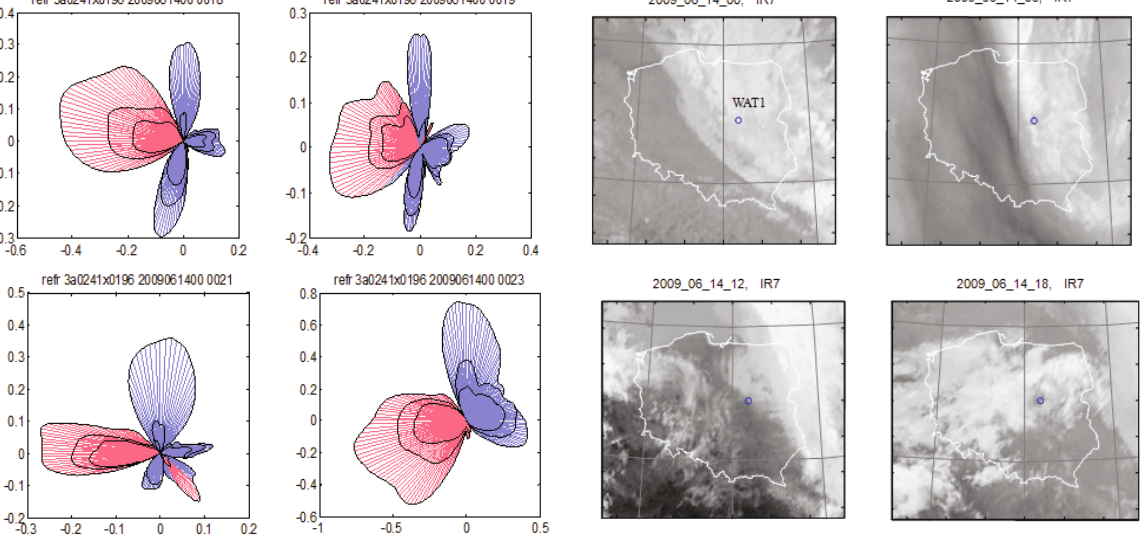

2009_06_14_18, IR7

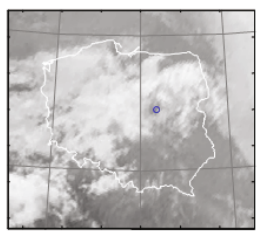

Fig. 11. Forecasted distributions of differences of slant delay $\Delta \tau_{r}^{s}=\Delta \tau^{s}-\overline{\Delta \tau^{s}}$ for 14 June 2009 . The weather condition related with an atmospheric front is presented in MSG 2 satellite images. Units: [m]. 

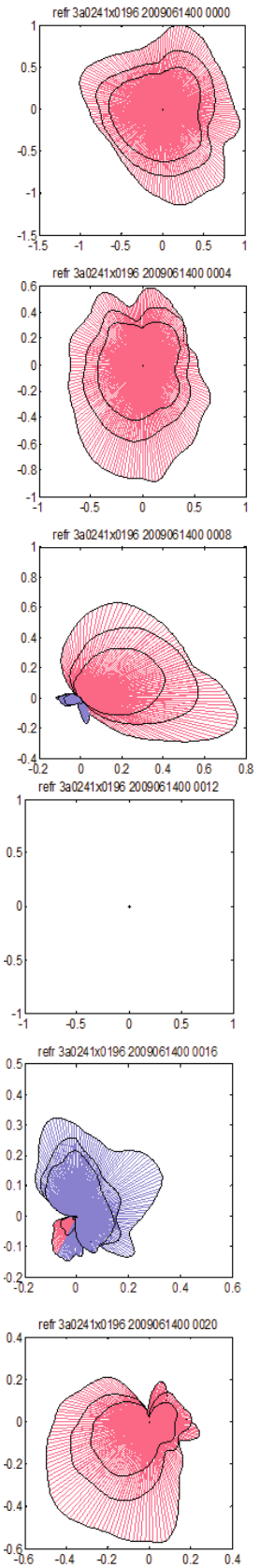
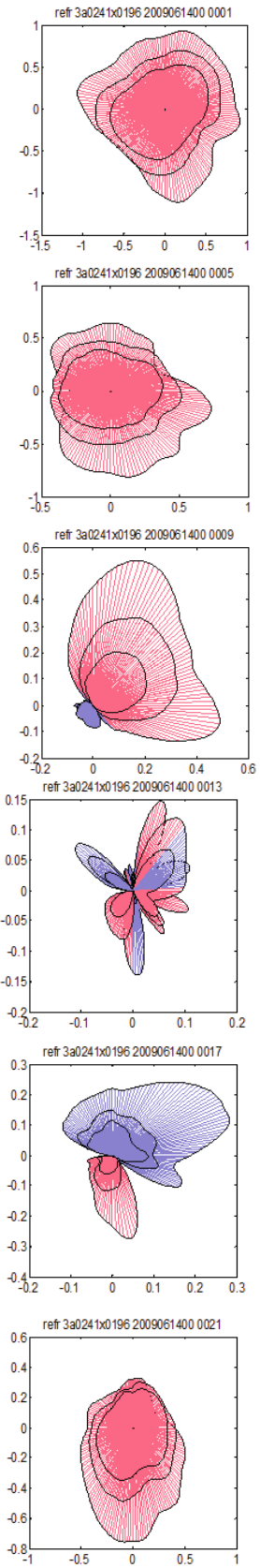
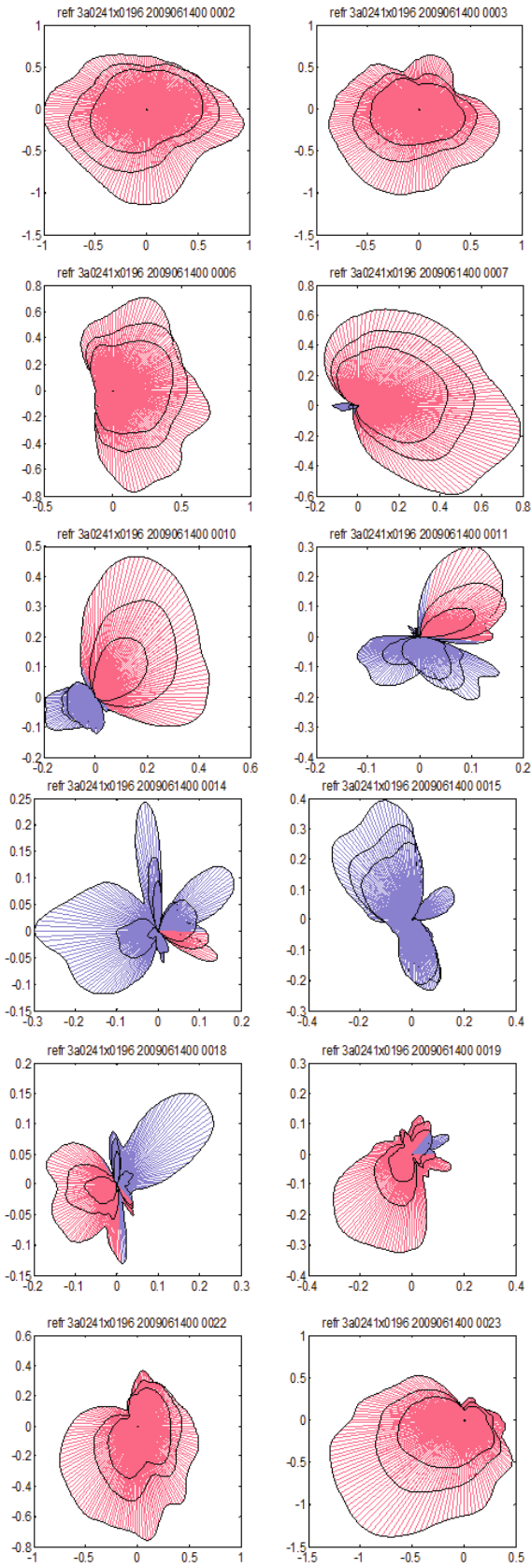

Fig. 12. Relative distributions of slant delay: $\Delta \tau_{r}^{s}=\Delta \tau^{s}-\Delta \tau^{s}\left(\cdot, t_{12}\right)$ for 14 June 2009. Units: [m]. 
Distributions presented in Figs. 8, 10, and 12 were also obtained using Eq. 12 but for differences $\Delta \tau^{s}(\cdot, \alpha)$ defined by

$$
\Delta \tau_{r}^{s}(\cdot, \alpha)=\Delta \tau^{s}(\cdot, \alpha)-\Delta \tau^{s}\left(\cdot, \alpha, t_{12}\right) .
$$

In this equation, $\Delta \tau^{s}\left(\cdot, \alpha, t_{12}\right)$ corresponds to the distribution of slant delay at 12 UTC. The plots of the type as in Fig. 8 present differences between the azimuth course at a specific hour of forecast and the course at 12 UTC for each of the elevation angles $\left(\varepsilon=3^{\circ}, 4^{\circ}, 5^{\circ}\right)$. Red/blue colour indicates greater/smaller values than the averaged discrete course with respect to azimuth of the slant delay $\Delta \tau^{s}$. Additionally, MSG 2 geostationary satellite images from selected spectral channels, meteorological elements fields for the computational surfaces of the mesoscale model (e.g., relative humidity), and vertical cross-sections indicating the conditions of the GPS signals propagation are included. Equations 12 and 13 do not use up all the possibilities of investigating the spatial distributions of slant delays. For example, $\overline{\Delta \tau^{s}}$ for given elevation angles may be azimuth daily series obtained as a result of averaging 24-hour forecasted courses. In this case, stronger variability of the distributions should be expected (Tables 1 and 2, and Fig. 13) because the daily average usually deviates significantly from the hourly averages used in Eq. 12. Other information related with the possibility of interpolating the delays for a receiver located in the neighbourhood of reference sites, e.g., ASG-EUPOS network, may be obtained by means of determination of spatial differences of delays between the network GPS sites for the same measurement epoch.

Analysis of the azimuth distributions of slant delays presented in Fig. 7 shows that they are similar. Such a situation, occurring for each hour of the forecast, indicates the azimuth stationarity of the weather conditions and at the same time the stationarity of the signal propagation conditions in the atmosphere. At this time, the WAT1 reference site was under cloud cover of an atmospheric front moving over Poland and in the beginning under influence of characteristic north-westerly advection of an air mass. Positive (red) and negative (blue) values of differences of delays slightly fluctuate, showing changes of the order of a decimetre. It is also observed that the directions of their extremes do not change significantly. Figure 8 shows a greater spatial and temporal variability of the delays distributions. In the first hours of the forecast, the differences are positive, reaching the values of the order of two decimetres for the elevation angle of $3^{\circ}$. It means an increase of the length of the signal propagation path in the atmosphere in comparison with the one for 12 UTC which determines the reference epoch. In the following hours, the signs and the directions of the differences change, while their am- 

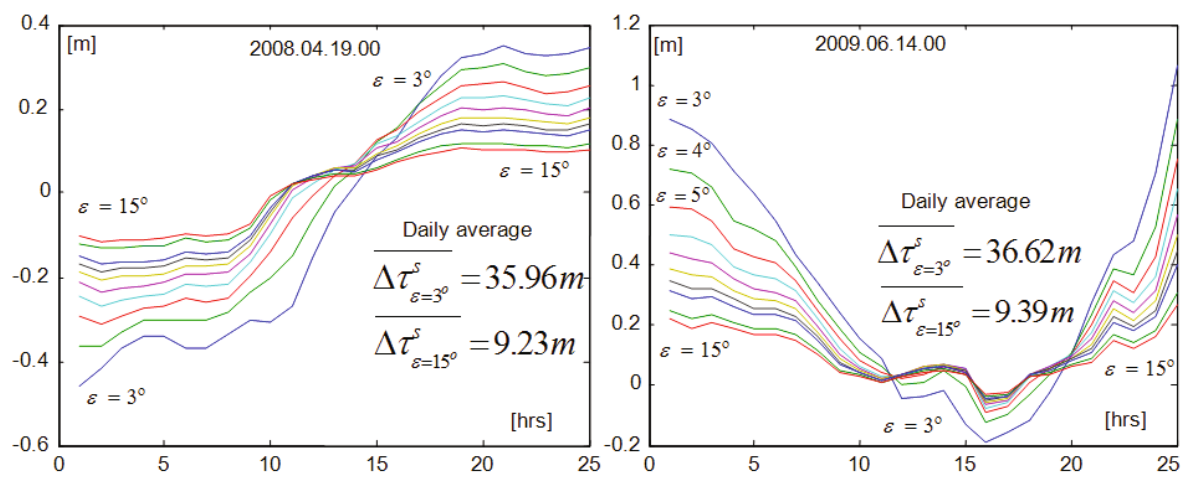

Fig. 13. Averaged azimuth differences of hourly and daily slant delays.

plitudes decrease below one decimetre. They are negative and much bigger (about $0.5 \mathrm{~m}$ ) after 16 hours of forecast which means improvement in the propagation properties of the atmosphere. On 19 April 2008 (Fig. 9), the values and the changes of directions of the slant delay differences are bigger than on 17 April 2008 all day long. The positive component in the westerly direction decreases starting at 00 UTC. At the same time, the south-easterly component appears and develops reaching about $0.5 \mathrm{~m}$. Then, in the afternoon hours of the forecast, the scenario is opposite. The plots in Fig. 10 indicate better transmission properties of the atmosphere in the first part of the day, and worsening of the properties in the following part. This is partly justified by cloud systems observed in the included satellite images in the vicinity of the WAT1 site. A similar situation, presented in Fig. 11, is observed for distributions investigated for comparison for 14 June 2009. In general, it may be concluded that positive differences (Figs. 7, 9, and 11) are usually correlated with directions indicating existence of a developed system of layer clouds, e.g., frontal system, which is related with areas of larger quantities of water vapour, while negative values correspond to GPS waves propagation through cloudless areas of the atmosphere. Distributions presented in Fig. 11 show (in comparison with the above-discussed ones) greater spatial and temporal variability of positive and negative directions of slant delay differences. They reflect in a good way the motion of cloud structures over the WAT1 site, as observed in satellite images. Investigation of angular characteristics (Fig. 12) shows that the differences values of the slant delays with respect to the reference delay of 12 UTC are almost $1 \mathrm{~m}$ in the first and last hours of the forecast. The distributions presented in Figs. 7 through 12 reflect relative spatial and temporal changes of slant delays resulting from the atmospheric conditions evolution in the vicinity of the WAT1 site. Similar characteristics may be obtained for all reference sites of the ASG-EUPOS 
system. Generally, they may be obtained for each point of the computational area of the mesoscale model which fulfils the criterion of feasibility of scanning (Eq. 12). It is said that they determine the conditions of microwaves propagation in the atmosphere, i.e., the GPS weather, for the considered area and time of the model data archiving. Function 12 used for investigating the heterogeneousness of the slant delays is based on the differences of the absolute courses and average courses corresponding to them. Application of such a method enables to detect the azimuth differences. However, it does not contain all the information. It is the case because the averages with respect to the azimuth determined for each hour of the forecast change in time. Figure 13 presents the temporal courses for various elevation angles referred to daily azimuth averages, i.e., computed using 24 values of hourly averages.

On 19 April 2008 the differences increased while on 14 June 2009 they decreased for some of the forecast time, and then they increased exceeding the initial values. In the analysed cases, the changes of the average delays for small elevation angles exceed $1 \mathrm{~m}$. The time courses (Fig. 13) also reflect well the character of temporal changes of differences of the distributions presented in Figs. 10 and 12. All the considered courses and angular distributions indicate existence of a usually neglected azimuth relation. The discrepancy from symmetry may be illustrated in a cylindrical system by means of transferring two-dimensional distributions (Figs. 7 through 12) to tridimensional space (Fig. 14). Horizontal cross-sections (contour lines) of the constructed surfaces correspond to the azimuth distributions for fixed elevation angles $\varepsilon$, while vertical cross-sections correspond to profiles of differences of slant delays for a selected value of azimuth $\alpha$.
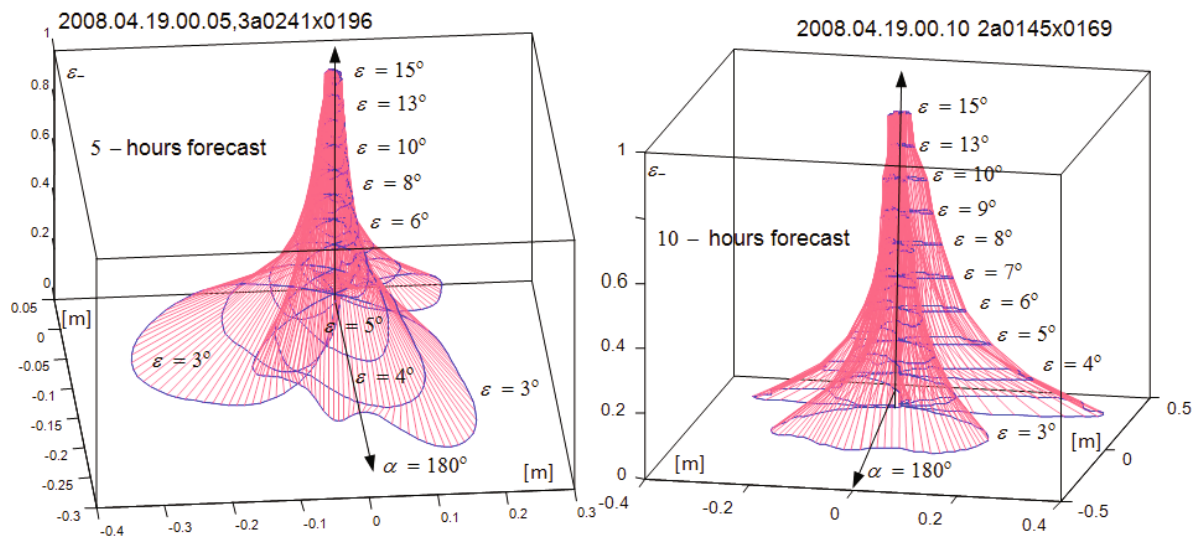

Fig. 14. Tridimensional anisotropic distributions of differences of slant delays and their hourly azimuth average values for the WAT1 reference site; $\alpha$ - azimuth, $\varepsilon_{-}-$ arbitrary value of the elevation angle $\left(\varepsilon_{-}=0 \rightarrow \varepsilon=0^{\circ}, \varepsilon_{-}=1 \rightarrow \varepsilon=90^{\circ}\right)$. 

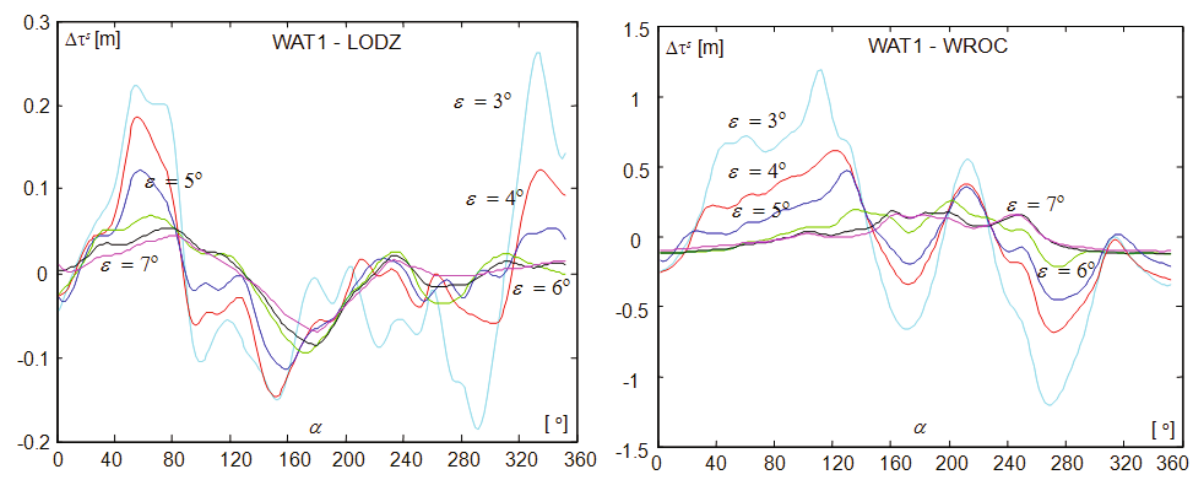

Fig. 15. Differences of slant delays between reference sites.

For appropriately large elevation angles $\left(\varepsilon>20^{\circ}\right)$, the function contour lines (Fig. 14) become circles (for $\varepsilon=90^{\circ}$ ) they convert to a point. It means that for measurements at such values of the cut-off angles, the azimuth asymmetry is negligible. Tridimensional discrete mapping functions may be obtained by adding to each of the contour lines of the selected distributions the hourly averages corresponding to them, and then dividing the values by zenithal delays. They are determined by the positions of GPS reference sites for measurement epochs related with subsequent hours of the forecast. These sets of functions (delays) enable us to analyse their spatial differences (Fig. 15).

The differences of slant delays (Fig. 15) between the WAT1 and the WROC reference sites are a few times larger than those for the WAT1 and the LODZ sites which means that the propagation conditions in the atmosphere are dramatically different for those sites.

\section{SUMMARY}

The conducted research proved that non-hydrostatic mesoscale model forecast data enable to model spatial and temporal heterogeneousness of atmospheric refraction fields which may be used for investigating and simulating GPS waves propagation in the atmosphere. The results of computational experiments showed that the slant delay is a function of the azimuth of observation for small values of elevation angles. This relation reflects spatial heterogeneousness of the atmospheric state including the distribution of humidity along the GPS signal path. It was observed that the azimuth asymmetry (anisotropy) of the slant delays reaches the value of $1 \mathrm{~m}$ and it may be even larger in unfavourable weather conditions. Defining differential angular characteristics enabled us to present and investigate the anisotropic distributions of the slant delay in a better way. Application of the mesoscale model 
data enabled us to determine them for various atmospheric conditions. A more precise explanation of heterogeneousness of the obtained distributions and their spatial and temporal evolution requires further research related with, e.g., increasing the number of computational levels of the weather models, especially in the lower, humid part of the troposphere.

Acknowledgments. This research was financed by the Faculty of Civil Engineering and Geodesy of the MUT statutory research funds.

\section{References}

Boehm, J., B. Werl, and H. Schuh (2006), Troposphere mapping functions for GPS and very long baseline interferometry from European Centre for MediumRange Weather Forecasts operational analysis data, J. Geophys. Res. 111, B2, B02406, DOI: 10.1029/2005JB003629.

Bogusz, J., M. Figurski, K. Kroszczyński, and K. Szafranek (2011), Investigation of environmental influences to the precise GNNS solutions, Acta Geodyn. Geomat. 8, 1, 5-15.

Bosy, J., W. Rohm, A. Borkowski, K. Kroszczyński, and M. Figurski (2010), Integration and verification of meteorological observations and NWP model data for the local GNSS tomography, Atmos Res. 96, 4, 522-530, DOI: 10.1016/j.atmosres.2009.12.012.

Dach, R., U. Hugentobler, P. Fridez and M. Meindl (2007), Bernese GPS software version 5.0, Astronomical Institute, University of Bern, Bern, Switzerland.

Figurski, M., M. Gałuszkiewicz, P. Kamiński, and K. Kroszczyński (2007), Mesoscale functions of GPS slant delay, Acta Geodyn. Geomater. 4, 4, 141144.

Figurski, M., M. Gałuszkiewicz, P. Kamiński, and K. Kroszczyński (2009), Mesoscale anisotropy of GPS slant delay, Bull. Geod. Geomatics 2, 99-110.

Hobiger, T., R. Ichikawa, Y. Koyama, and T. Kondo (2008), Fast and accurate raytracing algorithms for real-time space geodetic applications using numerical weather models, J. Geophys. Res. 113, D20, D20302, DOI: 10.1029/ 2008JD010503.

Hodur, R.M. (1997), The Naval Research Laboratory's Coupled Ocean/Atmosphere Mesoscale Prediction System (COAMPS), Mon. Weather Rev. 125, 7, 1414-1430, DOI: 10.1175/1520-0493(1997)125<1414:TNRLSC $>2.0 . C O ; 2$.

King, R.W., and Y. Bock (2005), Documentation for the GAMIT GPS processing software, Release 10.2, Massachusetts Institute of Technology, Cambridge, USA. 
Mendes, V.B. (1999), Modeling the neutral atmosphere propagation delay in radiometric space techniques, Ph.D. Thesis, Tech. Rep. 199, Department of Geodesy and Geomatics Engineering, University of New Brunswick, Fredericton, Canada.

Niell, A.E. (1996), Global mapping functions for the atmosphere delay at radio wavelengths, J. Geophys. Res. 101, B2, 3227-3246, DOI: 10.1029/ 95JB03048.

Niell, A.E. (2000), Improved atmospheric mapping functions for VLBI and GPS, Earth Planets Space 52, 10, 699-702, DOI: 10.1186/BF03352267.

Ralston, A. (1995), Introduction to Numerical Analysis, PWN, Warszawa (in Polish).

Rocken, C., S. Sokolovskiy, J.M. Johnson, and D. Hunt (2001), Improved mapping of tropospheric delays, J. Atmos. Ocean. Technol. 18, 7, 1205-1213, DOI: 10.1175/1520-0426(2001)018<1205:IMOTD>2.0.CO;2.

Urquhart, L., F.G. Nievinski, M.C. Santos (2011), Ray-traced slant factors for mitigating the tropospheric delay at the observation level, J. Geodesy 86, 2, 149-160, DOI: 10.1007/s00190-011-0503-x.

Wielgosz, P., J. Paziewski, A. Krankowski, K. Kroszczyński, and M. Figurski (2012), Results of the application of tropospheric corrections from different troposphere models for precise GPS rapid static positioning, Acta Geophys, 60, 4, 1236-1257, DOI: 10.2478/s11600-011-0078-1.

Zus, F., M. Bender, Z. Deng, G. Dick, S. Heise, M. Shang-Guan, and J. Wickert (2012), A methodology to compute GPS slant total delays in a numerical weather model, Radio Sci. 47, RS2018, DOI: 10.1029/2011RS004853.

Received 14 January 2015

Received in revised form 3 April 2015

Accepted 10 April 2015 